\title{
New screen predicts FSGS recurrence
}

Researchers have developed a novel antibody screen to estimate the risk of recurrence of focal segmental glomerulosclerosis (FSGS) - a major cause of accelerated graft loss-prior to kidney transplantation. "The inability of clinicians to predict FSGS recurrence is frustrating and adds to the organ shortage problem," explains author Minnie Sarwal.

To identify specific autoantibodies against glomerular proteins that might drive kidney injury in FSGS, Sarwal and colleagues profiled the pretransplant sera of 20 patients with primary FSGS against 9,000 human proteins. "Analysis of the highly coordinated autoantibody profiles in recurrent FSGS led us to uncover disease-causing proteins or target antigens," says Sarwal.

The researchers identified a panel of seven antibodies that could predict post-transplant FSGS recurrence in their cohort with $92 \%$ accuracy; anti-CD40 alone could predict recurrence with $78 \%$ accuracy. They posit that a simple blood test using their panel could enable risk-stratification of patients with primary FSGS awaiting transplantation.

Further work suggested an aetiologic role for perturbations of the CD40 antigen in recurrent

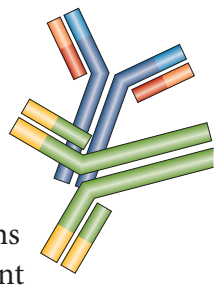
FSGS. "Anti-CD40 antibody is an exciting target; it may abrogate FSGS injury and might also be used as an induction immunosuppressive agent during organ engraftment," says Sarwal.

The researchers are now planning a trial of anti-CD40 antibody therapy in patients with FSGS. They hope that their work will enable personalized medicine for these patients, reduce morbidity, improve transplant safety and maximize allograft longevity.

Jessica K. Edwards

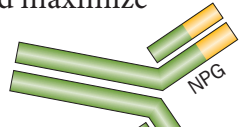

Original article Delville, M. et al. A circulating antibody panel for pretransplant prediction of FSGS recurrence after kidney transplantation. Sci. Transl. Med. 6, 256ra136 (2014) 